\section{$\underset{\substack{\text { hommes } \\ \text { \& migrations }}}{ }$}

\section{Hommes \& migrations}

Revue française de référence sur les dynamiques

migratoires

1302 | 2013

Le Japon, pays d'immigration?

\title{
Retour au pays natal le temps d'un voyage
}

"Nous, les exilés, à travers le voyage, on recherche qui on est."

\section{Hélène du Mazaubrun}

\section{(2) OpenEdition}

12 Journals

\section{Édition électronique}

URL : http://journals.openedition.org/hommesmigrations/2502

DOI : 10.4000/hommesmigrations.2502

ISSN : 2262-3353

\section{Éditeur}

Musée national de l'histoire de l'immigration

\section{Édition imprimée}

Date de publication : 1 avril 2013

Pagination : 148-151

ISBN : 978-2-919040-22-3

ISSN : $1142-852 X$

\section{Référence électronique}

Hélène du Mazaubrun, «Retour au pays natal le temps d'un voyage », Hommes \& migrations [En ligne], 1302 | 2013, mis en ligne le 12 septembre 2013, consulté le 22 septembre 2020. URL : http://

journals.openedition.org/hommesmigrations/2502; DOI : https://doi.org/10.4000/

hommesmigrations.2502 


\title{
COLLECTIONS
}

\section{RETOUR AU PAYS NATAL LE TEMPS D'UN VOYAGE}

"NOUS, LES EXILÉS, À TRAVERS LE VOYAGE, ON RECHERCHE QUI ON EST."

\begin{abstract}
HÉLÈNE DU MAZAUBRUN, chargée des collections, responsable de la Galerie des dons, Musée national de l'histoire et des cultures de l'immigration, CNHI
\end{abstract}

M athieu Do Duc naît à Saigon, au Vietnam, en 1958, au sein d'une famille de notables. Son grand-père était mandarin, son oncle, diplomate, ambassadeur aux Philippines au moment de la chute de Saigon. En 1965, avec le début de la guerre du Vietnam, son père, militaire de formation ayant participé au coup d'État, décide l'évacuation de sa famille vers la France. À cette période, le petit garçon de 7 ans ne réalise guère ce qui se passe et les bouleversements à venir qui en découleront'. Aujourd'hui, il se souvient davantage de l'arrivée que du départ. Comme pour beaucoup d'exilés, le déracinement dans un contexte violent ressurgit lorsqu'il évoque l'adaptation de sa famille en France. Ils ont débarqué à Marseille par avion. Pour la mère de Mathieu Do Duc, le choc culturel, comme l'éloignement de son pays et de sa famille, est terrible, au point de n'avoir d'autre choix que de repartir. "Ma mère ne se plait pas en France et retourne au Vietnam, pensant qu'on allait se retrouver (...). Sauf que mon père n'avait pas prévu que l'on reparte, il avait dit à ma mère: 'Vas-y, je te rejoins dans un an', mais en fait, il avait prévu de rester en France. (...) Ma famille a été scindée en deux." Voilà comment, par un tsunami historique, la guerre qui frappe le Vietnam, des destins familiaux basculent, se démantèlent. Le père s'installe donc à Marseille avec Mathieu et son frère, tandis que le reste de la fratrie part avec la mère vivre aux États-Unis, parce que le retour au Vietnam s'avère finalement impossible.

Adolescent, Mathieu Do Duc a pour unique préoccupation de revoir sa famille. En 1981, son père et son jeune frère partent aux États-Unis rejoindre la famille. Mathieu n'y reste qu'un an, car il retourne en France ensuite pour ses études. Aussi, lorsqu'il reçoit son titre de voyage ${ }^{2}$, est-ce un véritable soulagement: "Ce titre de voyage, c'était la promesse de revoir ma famille. C'était comme un vrai passeport pour la vie. En tant qu'exilé, à travers le voyage, on recherche qui on est." Cet objet si précieux, il en a fait don au musée en $2013^{3}$.

Devenu adulte, Mathieu avait pensé rejoindre sa mère et ses frères aux États-Unis. À Paris, pour préparer ce départ, il fait la rencontre de celle qui deviendra son épouse. Et ce sont finalement

\footnotetext{
1. La guerre du Vietnam (également appelée deuxième guerre d'Indochine) est une guerre qui a opposé de 1954 à 1975, d'une part, la République démocratique du Vietnam (ou Nord-Vietnam) avec son armée populaire vietnamienne, - soutenue matériellement par le bloc de l'Est et la Chine et le Front national de libération du Sud-Vietnam (ou Viet Cong) -, et, d'autre part, la République du Vietnam (ou SudVietnam), militairement soutenue par l'armée des États-Unis appuyée par plusieurs alliés (Australie, Corée du Sud, Thaïlande, Philippines). La guerre civile laotienne et la guerre civile cambodgienne sont des conflits annexes qui se sont déroulés en parallèle, et sur lesquels la guerre du Vietnam a eu un impact décisif. 2. Ce document administratif s'adresse aux personnes reconnues réfugiées ou apatrides par les autorités françaises ou bénéficiaires de la protection subsidiaire ; il permet de voyager à l'étranger. 3. Mathieu Do Duc est entré en contact avec le Musée national de l'histoire de l'immigration en 2012. Son témoignage a été recueilli au cours de l'année 2012 et 2013 par la personne chargée des collections ethnographiques.
} 
Don de Mathieu Do Duc, une photographie de son voyage retour après trente-neuf ans au Vietnam (C) MuséE NATIONAL DE L'HISTOIRE ET DES CULTURES DE L'IMMIGRATION, CNHI.

de nombreux allers-retours qu'il fera entre Marseille et les États-Unis, pays avec lequel il entretient un lien particulier : "Le pays, c'est la famille en réalité. Donc, la plupart de mes voyages se faisaient aux États-Unis."

\section{À la découverte d'un pays natal devenu étranger}

Un jour, alors que Mathieu Do Duc se retrouve au centre d'une chaîne intergénérationnelle entre sa mère et ses enfants..., le désir d'un "retour" au Vietnam, le temps d'un voyage, se fait sentir. C'est en 2004, après trente-neuf ans d'exil, que
Mathieu Do Duc décide de retrouver une part de son enfance laissée là-bas: "Mes souvenirs sont partis, se sont enfouis... j'ai comme un trou." Les traces de ce lien avec le pays natal et son enfance sont rares. Parmi elles, une lettre envoyée par son grand-père: "Saigon, le 31 juillet 1973. Mon cher petit-fils, profitant du passage de tonton Paul, je t'écris ces quelques lignes pour te remercier de tes bons sentiments à notre égard et, en même temps, pour vous adresser, à vous tous, à ton papa d'abord, à tes frères et à toi nos meilleurs vœux de bonne santé et de bonnes vacances. Et nous y joignons nos chaleureuses félicitations pour vos brillants succès à vos examens. Ici, nous pensons beaucoup à vous tous, nous attendons impatiemment le jour 
Don de Mathieu Do Duc, son titre de voyage

(c) Musée national de L'histoire et des cultures de l'immigration, CNHI.

où nous pourrons nous réunir de nouveau, comme autrefois. Ce qui nous console un peu de cette séparation forcée, c'est qu'elle vous permet à vous trois de faire de bonnes études et aussi, et surtout, la persévérance dans cette bonne voie pour mériter les sacrifices que vos parents s'imposent pour l'édification de votre avenir. Votre maman, vos soeurs et frère se portent bien, pépé Hoang aussi. Chez nous, santé bonne pour tout le monde. Tout le monde se souvient de vous tous dans les prières quotidiennes. Cher petit-fils, encore une fois merci de tes bons sentiments, bonne continuation, de bonnes vacances à vous tous, et félicitations renouvelées. Pépé Théophane Ngo Van Minh."

Mathieu Do Duc sait que les bribes de sa mémoire recomposent un pays rêvé et que la confrontation à la réalité sera comme les vers de Paul Éluard qu'il aime citer, lorsqu'il songe au Vietnam : "Un long trajet vers un grand pays aux serrures compliquées. Tout y rouille, saufle ciel4." Mathieu commence donc à se préparer : "Je savais que ça allait être le choc! Dans ma tête, j'ai essayé de me préparer comme un sportif." Et pour se protéger, il se munit d'un appareil photo. Comme pour filtrer son regard. De là, naîtra une part de lui-même : son activité de photographe. C'est donc le temps de ce voyage en 2004, que s'expriment trente-neuf ans de "tant d'années d'absence, de distance, d'indifférence ou de détachement feints, d'exil salutaire fatalement accepté, assumé au point de n'être plus en surface qu'un simple voyageur, un 'étrange étranger' en sa propre terre, sur son sol natal, en ses propres souvenirs".

\section{Le difficile retour de la mémoire}

Sur place, ce fut finalement la déception. "Lorsque je suis retourné au Vietnam, j'ai essayé de voir si les choses allaient émerger, et rien... ça a été une source de souffrance et de frustration. (...) Retourner, c'est repartir sur les territoires lointains d'une mémoire en jachère, à contre-courant d'une terre en devenir, d'un peuple en marche forcée à l'assaut de son inéluctable destin, dans son défi contre le poids d'un passé peuplé de fantômes d'une guerre sans fin. Retourner, c'est caresser le rêve d'une tortue géante sans âge, dans un frêle esquif de nuages et d'illusions perdues dans le fracas des vagues. C'est parsemer de souvenirs d'enfant une plage sans cesse balayée par la houle des jours terriblement ordinaires. Remuer avec résignation et douleur ces galets enfouis dans la naissance d'un sourire, d'un geste, d'une odeur, d'une sensation, d'un regard, 
d'un vide, d'un rien qui réveilleront en nous le bruissement imperceptible d'une cicatrice qui affleure. (...) On s'imagine capable de dominer et on est submergé, chahuté. On pense contrôler et on est malmené. On espère être maitre et on est voué à subir la loi du vide, la fureur de l'oubli, le poids de la différence, les assauts de l'indifférence. Alors on se raccroche en plein naufrage, en pleine tempête, à son appareil photo, on se réfugie tant bien que mal derrière l'objectif pour s'arracher les yeux et le coeur pour quelques images qui surprendront ou au mieux suspendront, peut-être, l'idée qu'on se faisait d'un pays qui n'est plus celui d'où l'on vient mais bien celui d'où l'on naît, celui d'où l'on n'est plus que par fragments de mémoire, par éclats, par indices, imperceptibles fêlures de l'âme. Désespérément, on fouille chaque regard, chaque visage, chaque attitude, à l'affût d'une lueur, d'un rayon même infimes, qui éclaireront ces nuits à venir où le sommeil, on le sent bien, jouera à cache-cache, à qui perd gagne. Car c'est certain, l'autre est bien un 'je' en qui nous désirons projeter une indulgence sans partage tant il est vrai qu'il sera à jamais notre vrai-semblable." En plus de l'humour et de la distance dont il fait preuve pour dépasser et dédramatiser des situations gênantes, Mathieu Do Duc réalise des photographies en noir et blanc. Pourquoi ne pas utiliser la couleur ? Est-ce pour lutter contre des clichés chatoyants, exotiques, et l'idée des vacances? Pour lui, il faut "vider sa tête des images d'Épinal, de nostalgie post-indochinoise, de visions idylliques des brochures touristiques pourêtres en mal d'exotisme. Ne pas s'encombrer de cet 'asiatisme' de bazar empesé trop parfaitement ajusté à l'air du temps". Le noir et blanc sert-il à renouer avec l'enfance ; avec le passé ? Ce sont des captations de la vie sous la pluie, des personnes âgées accroupies, des enfants qui s'amusent dans un parc. Sa photographie préférée, son don au musée, représente un enfant suspendu dans le vide au-dessus de l'eau. On ne sait pas s'il saute en arrière ou en avant, pied gauche et mains dans un sens, pied droit et tête dans l'autre. Au développement de la pellicule, un filtre de lumière scinde l'image, plus foncée à gauche, plus claire à droite. Est-ce une image en miroir d'une mémoire - flottant abstraitement dans les airs du pays natal quitté trop tôt - qui ne se sait pas comment elle retombera sur la terre du Vietnam ? L'enfant retombera-t-il dans l'eau ? Ses cheveux sont encore mouillés. Rappelons que "Vietnam" veut dire "eau", en écho aux rivières et ruisseaux qui le sillonnent et à son très long littoral. Mathieu Do Duc, comme cet enfant, prendil impulsion sur le passé, comme s'il fallait plier les genoux vers ses racines pour mieux sauter? "Retourner aux sources peut-être pour renaitre à l'envi, aller plus avant vers d'autres horizons de montagnes bleues et d'eaux vertes... Embrasser goulûment chaque larme de vie et chaque goutte d'eau, de rosée, qui s'offrent à nos lèvres pour étancher la soif des heures, des jours, des mois et des errances à venir."

Son voyage aura duré quelques semaines et il lui faudra encore du temps pour s'en remettre, car le retour est souvent une façon de se découvrir et de construire sa propre identité. "Je me suis dis : Mince! Tu viens chercher tes racines et tu vois que tu n'as aucune connexion. J'ai fait beaucoup de photos pour me réapproprier mon pays, puisque ma parole était fermée, je n'avais pas le vocabulaire ; cétait ma façon de m'exprimer... C'est une histoire de deuil... à la fin de ce voyage, tu sais que tu n'es pas vietnamien, tu sais que tu n'es pas chez toi. (...) Un pays d'où l'on ne serait en définitive jamais parti mais dont on serait désormais délivré, absous de je ne sais quelle faute originelle. Se réconcilier avec l'eau, avec les larmes de ma mère. (...) Garder en soi la brûlure toujours vive d'un retour inachevé à jamais et continuer de sourire malgré tout, seule réponse au temps qui passe. (...) Retourner au pays, c'est rentrer non pas chez soi, mais en soi (...). Retourner, c'est labourer à nouveau pour mettre en semence les graines d'un futur sans remords, sans doutes ni regrets." 